\title{
Magnetic Resonance Imaging in Diagnosis of Complications of Renal and Ureteral Injuries in Different Periods of Traumatic Disease
}

\author{
Zavazhat M. Magomedova*, PGS; Elena A. Egorova, PhD, ScD; \\ Dmitry A. Lezhnev, PhD, ScD; Margarita V. Smislenova, PhD, ScD \\ Moscow State University of Medicine and Dentistry named after A. I. Evdokimov \\ Moscow, the Russian Federation
}

\begin{abstract}
The aim of this study was to evaluate the effectiveness of MRI in diagnosing combined renal and ureteral injuries at different periods of traumatic disease (TD).

Methods and Results: We analyzed the results of diagnostics and treatment of 139 patients (80 women and 59 men) with renal and ureteral injuries aged between 18 and 72 years. There were 67(48.2\%) patients in the period of acute reaction to trauma, $40(28.8 \%)$ patients with early manifestations, and $32(23 \%)$ patients in the period of late manifestations. In $127(91.4 \%)$ patients, an urgent plain abdominal X-ray was performed without any preliminary preparation. USI of the abdominal and retroperitoneal space was performed in 108(77.7\%) patients in the stage of the primary assessment of renal injury as it was a rapid non-invasive investigation. A whole-body MSCT was performed in 131(94.2\%) patients, using the nonionic contrast agents Ultravist (350mg I/ $\mathrm{ml}$ ) and Omnipaque (350 $\mathrm{mg} \mathrm{I} / \mathrm{ml}$ ). MRI was performed in $125(89.9 \%$ ) patients, including cases of pregnancy and a medical history of allergies. Contrast-enhanced MSCT had a high diagnostic efficiency in assessing complications in kidney and ureteral injuries at different periods of TD (accuracy of $89.2 \%$ for acute reaction, $88.8 \%$ for early manifestations, and $89.5 \%$ for late manifestations). MRI of the kidneys and ureters was indicated in periods of early and late manifestations of TB to detect renal complications in cases with a discrepancy between clinical manifestations and the results obtained by ultrasound and MSCT (accuracy of $87.5 \%$ for early manifestations and $89.9 \%$ for late manifestations).(International Journal of Biomedicine. 2021;11(3):342-345.)
\end{abstract}

Key Words: magnetic resonance imaging $\bullet$ multislice computed tomography $\bullet$ trauma $\bullet$ kidney $\bullet$ ureter

For citation: Magomedova ZM, Egorova EA, Lezhnev DA, Smislenova MV. Magnetic Resonance Imaging in Diagnosis of Complications of Renal and Ureteral Injuries in Different Periods of Traumatic Disease. International Journal of Biomedicine. 2021;11(3):342-345. doi:10.21103/Article11(3)_OA10

\section{Abbreviations}

ADC, apparent diffusion coefficient; CTU, computed tomography urography; MSCT, multislice computed tomography; MRI, magnetic resonance imaging: MRU, magnetic resonance urography; TD, traumatic disease; USI, ultrasound investigation; UT, urinary tract.

\section{Introduction}

Trauma still remains one of the most frequent causes of mortality in both Western countries and the Russian Federation. According to various authors, retroperitoneal hemorrhage

*Corresponding author: Zavazhat M. Magomedova, PGS. Department of Radiodiagnostics, Moscow State University of Medicine and Dentistry named after A. I. Evdokimov, Moscow, Russia.E-mail: ros-trum1@yandex.ru is detected in one-quarter of patients with closed abdominal trauma. ${ }^{(1)}$ In the general structure of traumatism, the cases of combined renal injuries are 1.0 to $8.0 \%$. The frequency of complications in patients with renal injury reaches $12 \%$ to $84 \%$. $^{(2)}$

Despite the diagnostic measures and therapeutic possibilities, disability and mortality among the injured with complications of combined kidney injury are still quite high. The urgency of the problem of diagnosing kidney damage in combined injury is determined by many factors. The most 
important of these include their severity, difficulty in diagnosis and treatment, and a high percentage of the mortality rate. ${ }^{(3,4)}$ The highest difficulty is the diagnosis of renal complications, due to the blurring of clinical manifestations, the difficulty of interpreting the results obtained, and the limitations of a number of diagnostic investigations in patients with severe trauma. In the later periods of traumatic disease (TD), the condition of the victims allows, in most cases, carrying out the necessary examinations without significant restrictions, which in turn expands the possibilities for the timely diagnosis of complications. ${ }^{(2)}$

The X-ray examination traditionally starts from plain $\mathrm{X}$-ray. However, in patients in serious condition, the required preparation before the examination is not carried out, which complicates the interpretation of plain radiographs and results of intravenous urography in cases of paresis of the colon or a decrease in renal excretory function on the affected side. ${ }^{(5)}$ Ultrasound investigation (USI) is one of the modern, affordable methods for assessing the condition of the urinary tract (UT) and is carried out according to a standard protocol. USI has a number of undeniable advantages, such as not exposing the patient to radiation, accessibility, and non-invasiveness; but it also has significant disadvantages - limitation of visualization in obesity, flatulence, low sensitivity in visualizing the ureter, which reduces the diagnostic value of the method. ${ }^{(6)}$

Currently, a whole-body MSCT is the first-line diagnostic tool in patients with multiple injuries. In comparison with other radiation methods, computed tomography urography (CTU) has the greatest diagnostic value in determining the causes of hematuria and urinary obstruction. ${ }^{(7)}$ MRI has a high potential for diagnosing acute renal and urinary tract injury in different periods of TD. MRI examination allows obtaining images of the urinary tract comparable in their informative value to MSCT in determining perirenal hematomas, assessing the depth of damage and viability of the renal parenchyma, and better visualizing of previously existing pathological changes in the organs of the urinary system. ${ }^{(8)}$

The aim of this study was to evaluate the effectiveness of MRI in diagnosing combined renal and ureteral injuries at different periods of TD.

\section{Material and Methods}

We analyzed the results of diagnostics and treatment of 139 patients ( 80 women and 59 men) with renal and ureteral injuries aged between 18 and 72 years. There were 67(48.2\%) patients in the period of acute reaction to trauma, $40(28.8 \%)$ patients with early manifestations, and $32(23 \%)$ patients in the period of late manifestations. All patients with suspected renal and ureteric damage were examined and treated according to the standard scheme, depending on the nature of the dominant pathology.

In $127(91.4 \%)$ patients, an urgent plain abdominal $\mathrm{X}$-ray was performed without any preliminary preparation, using the Axiom Luminos TF (Siemens). A plain abdominal $\mathrm{X}$-ray in frontal projection was performed with the patient in standing position, or in cases where the patient could not stand behind the screen of the X-ray apparatus, the study was performed in a horizontal position. The radiation dose was of $0.033-0.21 \mathrm{mSv}$.

USI of the abdominal and retroperitoneal space was performed in $108(77.7 \%$ ) patients in the stage of the primary assessment of renal injury as it was a rapid non-invasive investigation. The standard time for complete examination of the kidneys and ureters was 15 minutes. Visualizing unexpanded ureters by USI was difficult; the quality of the investigation largely depended on the patient's physical constitution.

A whole-body MSCT was performed in 131(94.2\%) patients on a GE Optima CT660 apparatus according to the "Polytrauma" program, using the nonionic contrast agents Ultravist (350mg I/ml) and Omnipaque (350mg I/ml). The radiation dose was of $10-18 \mathrm{mSv}$. The scanning area included: a) Skull and brain, cervical spine with the capture of the upper thoracic spine (up to the level of the ThIV vertebral body) - native scanning; b) Chest and abdominal cavities with intravenous contrast enhancement (with an assessment of the condition of internal organs, blood vessels, peripheral skeleton, and spine at the scan level). Unlike USI, MSCT is more independent of the operator; however, it involves ionizing radiation and requires intravenous injection of a contrast agent. If signs are detected of kidney injury (clinical or echographic) and stable hemodynamics, patients are recommended to perform the contrast-enhanced MSCT, but it requires puncture of the peripheral vein and multiphase scanning, taking approximately 10-15 minutes.

MRI has great potential for examining patients with UT injuries. MRI was performed in 125(89.9\%) patients, including cases of pregnancy and a medical history of allergies. Image acquisitions were performed using Siemens Magnetom Harmony MRI Scanner. Each patient was scanned supine on the examination couch with his or her feet first, with a coil covering the diaphragm to iliac crest. The routine imaging protocol of the kidney included a coronal/sagittal T2 half Fourier single-shot turbo spin-echo sequence (HASTE) and a native coronal 3D gradient echo pulse T1-weighted (FLASH) sequence. In addition, the patients received a native, balanced steady-state free precession T2 mapping sequence (T2-prepared single-shot TrueFISP) in coronal plane, short inversion time inversion recovery (STIR) sequence, as well as DWI with ADC values.

\section{Results and Discussion}

All patients were divided into groups according to the type of renal and ureteric injury: combined renal injuries $(125 / 89.9 \%)$, acute ureteral injuries $(11 / 7.9 \%)$, and isolated kidney injury $(3 / 2.2 \%)$. In case of damage, the clinical manifestations were nonspecific and the radiation signs were poor, which makes the diagnosis difficult. In 125(89.9\%) patients, renal and ureteral damages were combined with other localizations: ribs and thoracic organs (42/33.6\%); traumatic brain injury (30/24\%); pectoral girdle bones, pelvic bones and free limbs $(24 / 19.2 \%)$; the abdominal cavity organs $(17 / 13.6 \%)$; spinal-cord injury $(12 / 9.6 \%)$.

The greatest difficulty was renal damage in the acute and early periods of TD manifested by the blurred clinical image 
against a background of shock. Credible features of the kidney injuries were determined by the results of MSCT, which gave an opportunity to establish the severity of the injury (Fig. 1).

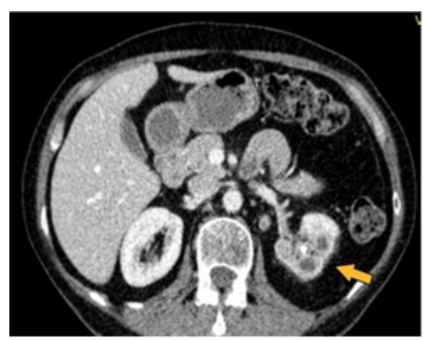

(a)

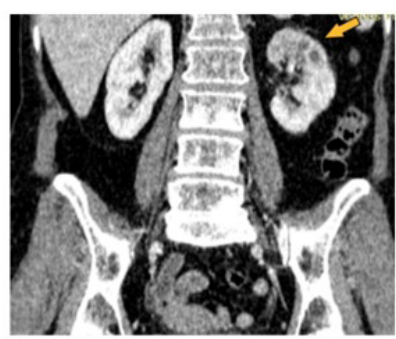

(b)
Fig. 1. Contrast-enhanced MSCT ( $a-b)$ of the abdominal cavity and retroperitoneal space (multiplanar reconstruction) of a 62-year-old patient on Day 4 after trauma: abscesses in the upper pole of the left kidney as a hypodense area with indistinct contours (arrows).

MRI was performed in patients whose MSCT data were ambiguous and did not correspond to clinical and laboratory parameters. MRI has a high potential in the diagnosis of acute renal and urinary tract injury in different periods of TD and allows us to obtain images of the urinary tract comparable in its informational content to MSCT in determining the perirenal hematomas, assessing the depth of damage and viability of the renal parenchyma, providing better visualization of previously existing pathological changes of the urinary system organs while there is no radiation exposure to the patient and there is a natural tissue contrast without the need for the mandatory usage of intravenous contrast (Fig. 2).

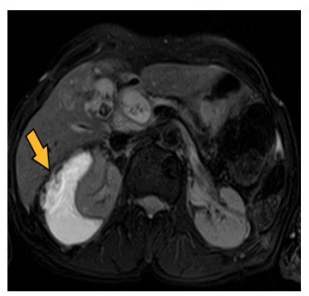

(a)

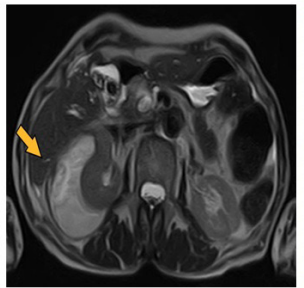

(b)

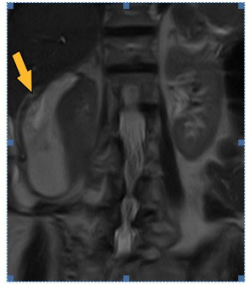

(c)
Fig. 2. Native MRI of the abdominal cavity and retroperitoneal space (multiplanar reconstructions) of a 62-year-old patient on Day 4 after trauma: subcapsular hematoma in the upper, middle, and lower third of the right kidney (arrows).

There were 26(20.3\%) patients operated on in the acute period of TD. Nephrectomy was performed in 7(5.47\%) cases with involvement of more than $25 \%$ of the kidney parenchyma in the pyo-inflammatory process; drainage of the retroperitoneal space - in $8(6.25 \%)$ cases; organ-preserving operations (wound closure or kidney resection) - in 11(8.6\%) cases. Besides surgical interventions for the renal and ureteral injuries, there were indicated purulent complications for surgical treatment (48/37.5\%).

Modern technologies and MRI software allowed us to eliminate many of the previous drawbacks connected to breathing artifacts. Optimizing protocols to reduce the total time of investigation to $10-15 \mathrm{~min}$, using the necessary and sufficient program set in combination with the high differentiation of soft tissues, changed the place of MRI in the diagnostic algorithm in patients with renal damages. MRI has a high informational content and absence of ionizing radiation that makes this method demanded among pregnant women and the injured persons for whom contrast-enhanced MSCT is contraindicated (Fig.3). The MRI study revealed purulent inflammation of the kidneys in 3 pregnant patients who could not undergo CTU. MRI diagnostic viability is commeasurable with the contrast-enhanced MSCT. It doesn't have an ionizing impact and mostly does not need injection of any contrast agents. In our study, the duration of the investigation using a short protocol reached $15 \mathrm{~min}$, which is similar to MSCT examination, taking to account the time for injection and catheterization of the peripheral vein, the native scanning, and multiphasic post-contrast scanning. The implementation of MRI allowed us to reduce radiation exposure and to increase the economic effectiveness of diagnosis by reducing the investigation costs. MRI, in combination with high indicators of efficiency and low costs and in contrast to intravenous contrast-enhanced MSCT, allows using this method for obtaining complete and sufficient information in the diagnosis of renal and ureteral injuries without significant restrictions (Fig. 4).

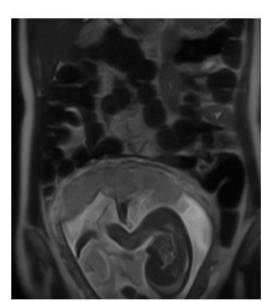

(a)

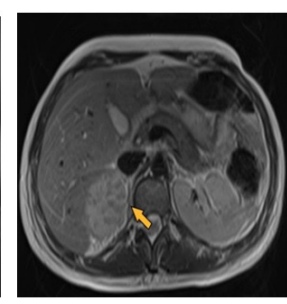

(b)

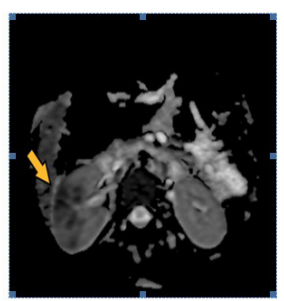

(c)
Fig. 3. MRI of a pregnant woman (21-22 weeks): a) - T2-WI in the coronary plane, the uterus is enlarged according to the period of gestation, a single fetus in the cephalic presentation in the uterine cavity; b) MRU: thickening of the parenchyma with apostems in the cortical layer (arrows), c) DWI and ADC: foci of diffusion limitation correspond to multiple apostems (arrows).

The developed short MRI protocol enables us to reach high diagnostic efficiency in patients with renal and ureteral injuries during different periods of TD and accurate detection of pathological changes in the abdominal cavity and retroperitoneal space. Taking into account the above mentioned, we calculated the scores of sensitivity (SE), specificity (SP), and accuracy (AC). The results presented in Table 1 showed that all the investigative methods demonstrate an increase in accuracy in detecting traumatic changes in the kidneys and ureters from the period of acute reaction to injury up to the period of late onsets of TD.

\section{Conclusion}

1. Contrast-enhanced MSCT has a high diagnostic efficiency in assessing complications in kidney and ureteral injuries at different periods of TD (accuracy of $89.2 \%$ for acute reaction, $88.8 \%$ for early manifestations, and $89.5 \%$ for late manifestations). 
Table 1.

The diagnostic efficiency of the investigative methods in detecting renal and ureteral injuries at different periods of $T D$

\begin{tabular}{|l|l|c|c|c|}
\hline \multirow{2}{*}{$\begin{array}{c}\text { Diagnostic } \\
\text { methods }\end{array}$} & TB periods & \multicolumn{3}{|c|}{ Indicators of diagnostic efficiency,\% } \\
\cline { 2 - 5 } & & $\mathrm{Se}$ & $\mathrm{Sp}$ & $\mathrm{Ac}$ \\
\hline \multirow{2}{*}{$\begin{array}{l}\text { Clinical and } \\
\text { laboratory } \\
\text { examination }\end{array}$} & acute reaction & 61.2 & 56.1 & 57.5 \\
\cline { 2 - 5 } & early onset & 66.4 & 61.2 & 64.1 \\
\cline { 2 - 5 } & late onset & 86.3 & 82.7 & 85.6 \\
\hline \multirow{4}{*}{ Plain X-ray } & acute reaction & - & - & - \\
\cline { 2 - 5 } & early onset & 13.7 & 15.9 & 13.1 \\
\cline { 2 - 5 } & late onset & 17.8 & 44.7 & 25.2 \\
\hline \multirow{3}{*}{ USI } & acute reaction & 68.3 & 35.9 & 46.7 \\
\cline { 2 - 5 } & early onset & 71.9 & 64.7 & 72.0 \\
\cline { 2 - 5 } & late onset & 89.9 & 82.7 & 86.3 \\
\hline \multirow{3}{*}{ MSCT } & acute reaction & 92.0 & 84.9 & 89.2 \\
\cline { 2 - 5 } & early onset & 92.7 & 86.3 & 88.8 \\
\cline { 2 - 5 } & late onset & 94.2 & 84.8 & 89.5 \\
\hline \multirow{3}{*}{ MRI } & acute reaction & - & - & - \\
\cline { 2 - 5 } & early onset & 88.5 & 80.6 & 87.05 \\
\cline { 2 - 5 } & late onset & 92.1 & 87.8 & 89.9 \\
\hline
\end{tabular}

2. MRI of the kidneys and ureters is indicated in periods of early and late manifestations of TD to detect renal complications in cases with a discrepancy between clinical manifestations and the results obtained by ultrasound and MSCT (accuracy of $87.5 \%$ for early manifestations and $89.9 \%$ for late manifestations).

3. The MRI protocol with both conventional spinecho and STIR sequences allows achieving high diagnostic efficiency.

\section{Competing Interests} interests.

The authors declare that they have no competing

\section{References}

1. Smolyar AN. [Blunt abdominal trauma. Kidneys injury. Part 3]. Pirogov Russian Journal of Surgery. 2016;(6):4-13. [Article in Russian].

2. Komyakov BK, Soroka IV, Savello VE, Shanava GSh, Basek IV. [Features of the diagnosis of renal complications of concomitant renal injury in the acute and early periods of traumatic diseases]. Biomed J. 2011;12:1467-1477. [Article in Russian].

3. Perkins ZB, Captur G, Bird R, Gleeson L, Singer B, O'Brien B. Trauma induced acute kidney injury. PLoS One. 2019 Jan 25;14(1):e0211001. doi: 10.1371/journal. pone.0211001.

4. Eriksson $\mathrm{M}$, Brattström $\mathrm{O}$, Mårtensson $\mathrm{J}$, Larsson E, Oldner A. Acute kidney injury following severe trauma: Risk factors and long-term outcome. J Trauma Acute Care Surg. 2015 Sep;79(3):407-12. doi: 10.1097/TA.0000000000000727. 5. Pushkar DYu, Kasyan GR. Errors and complications in urogynecology. M.: GEOTAR-Media, 2017. [In Russian].

6. Wieczorek AP, Woźniak MM, Tyloch JF. Errors in the ultrasound diagnosis of the kidneys, ureters and urinary bladder. J Ultrason. 2013 Sep;13(54):308-18. doi: 10.15557/ JoU.2013.0031.

7. Vasiliev AYu, Vinogradova OA. [Multislice computed tomography in the diagnosis of postoperative changes in the ureter]. Radiology and Practice. 2017;2(62):6-13. [Article in Russian].

8. Martínez-Piñeiro L, Djakovic N, Plas E, Mor Y, Santucci RA, Serafetinidis E, Turkeri LN, Hohenfellner M; European Association of Urology. EAU Guidelines on Urethral Trauma. Eur Urol. 2010 May;57(5):791-803. doi: 10.1016/j. eururo.2010.01.013. 